Proceedings of the European Conference Physics of Magnetism 2011 (PM'11), Poznań, June 27-July 1, 2011

\title{
Magnetic Field Effects in Frustrated Low-Dimensional Magnets
}

\author{
B. Schmidt, M. Siahatgar And P. Thalmeier
}

Max-Planck-Institut für Chemische Physik fester Stoffe, 01187 Dresden, Germany

We investigate the frustrated two-dimensional $S=1 / 2$ next nearest neighbor anisotropic Heisenberg antiferromagnet on a square lattice as described by the $J_{1 a, b}-J_{2}$ model. We use spin-wave theory and exact diagonalization for finite tiles including a new method for the finite size scaling procedure. We present results obtained from the extension of our numerical method to finite magnetic fields as well as from spin-wave theory. The induced uniform and the staggered moment in the antiferromagnetically ordered phases in the presence of a magnetic field are calculated. They deviate strongly from classical behaviour depending on frustration ratio $J_{2} / J_{1 a, b}$ and the $J_{1 a, b}$ exchange anisotropy. The magnetization becomes strongly nonlinear and is suppressed from the classical value. This is due to enhanced quantum fluctuations already at moderate frustration.

PACS: 75.10.Jm, 75.30.Cr, 75.30.Ds

\section{Introduction}

We present recent results from our investigation of the frustrated two-dimensional $S=1 / 2$ next nearest neighbor anisotropic Heisenberg antiferromagnet on a square lattice as described by the $J_{1 a, b}-J_{2}$ model [1, 2]. This model has a number of realizations in layered $\mathrm{V}^{4+}$ compounds $[3-5]$. Combining the results of several experimental investigations, the determination of the location of these compounds in the phase diagram was possible [6-8]. It was eventually found that all known compounds are lying in the region of columnar antiferromagnetic (CAF) order, characterized by an ordering vector $\boldsymbol{Q}=(\pi, 0)$ or $(0, \pi)$. Strictly speaking, the $\mathrm{V}^{4+}$ compounds all have slight orthorhombic distortions, leading to a spatial anisotropy in the nearest-neighbor exchange constants $J_{1 a}$ and $J_{1 b}$ along the respective crystallographic directions.

Recently, results from inelastic neutron scattering (INS) on the low-energy excitations of the 122 Fe pnictides have shown that these can also be described by a local-moment model with nearest- and next-nearest neighbor exchange integrals, despite the metallic nature of these compounds [9-12]. Here, a spatial anisotropy of the exchange parameters has been introduced, too.

INS results also show that in the Fe pnictides, well-defined spin excitations exist in the whole Brillouin zone, which suggests a local-moment picture for the magnetic excitations to be applicable. The experimentally observed size of the ordered moment is strongly reduced compared to predictions from density-functional theory. In this report, we summarize our results on the anisotropic frustrated two-dimensional $S=1 / 2$ Heisenberg model on the square (or better rectangular) lattice. Within this model, it seems natural to investigate to what extent frustration can serve as an origin for the observed moment reduction.

\section{Theory}

The Hamiltonian we discuss has the form

$$
H=\sum_{\langle i j\rangle} J_{i j} \boldsymbol{S}_{i} \boldsymbol{S}_{j}-g \mu_{\mathrm{B}} H \sum_{i} S_{i}^{z}
$$

where $J_{i j}=\operatorname{diag}\left(J_{i j}^{\perp}, J_{i j}^{\perp}, J_{i j}^{z}\right)$, and $J_{i j}=J_{1 a}$ or $J_{1 b}$ if $i$ and $j$ mark nearest-neighbor sites along the crystallographic $a$ and $b$ directions, respectively, $J_{i j}=J_{2}$ if $i$ and $j$ denote next-nearest neighbors, and the sum runs over all nearest- and next-nearest neighbor bonds. The magnetic field $\boldsymbol{h}=g \mu_{\mathrm{B}} \boldsymbol{H}$ points along the $z$ direction in spin space. On each site $i$, we introduce a local coordinate system, where the $z$ axis is oriented parallel to the local magnetic moment, and express the spin operator products in Eq. (1) in these coordinates.

For the classical ground-state energy, we get

$$
E_{\mathrm{cl}}=N S^{2}\left[J_{\perp}(\boldsymbol{Q})-A(0) \cos ^{2} \Theta_{\mathrm{c}}\right],
$$

where $J_{\perp}(\boldsymbol{Q})$ is the Fourier transform of the exchange constants perpendicular to the magnetic field, $\Theta_{\mathrm{c}}$ is the canting angle of the spins with respect to the magnetic field given by $\cos \Theta_{\mathrm{c}}=h /[2 S A(0)]$, and

$$
\begin{aligned}
& A(\boldsymbol{k})=J_{z}(\boldsymbol{k})+\frac{1}{2}\left[J_{\perp}(\boldsymbol{k}+\boldsymbol{Q})+J_{\perp}(\boldsymbol{k}-\boldsymbol{Q})\right] \\
& \quad-2 J_{\perp}(\boldsymbol{Q}) .
\end{aligned}
$$

Minimizing $E_{\mathrm{cl}}(\boldsymbol{Q})$ with respect to $\boldsymbol{Q}$ yields the ordering vector $Q$.

Within the framework of linear spin-wave theory, we expand the Hamiltonian around its classical limit up to first order in $1 / S$. The result is

$$
\mathcal{H}=E_{\mathrm{cl}}+E_{\mathrm{zp}}+S \sum_{\boldsymbol{k}} E(h, \boldsymbol{k}) \alpha_{\boldsymbol{k}}^{\dagger} \alpha_{\boldsymbol{k}},
$$

where $E_{\mathrm{cl}}$ is given by Eq. (2),

$$
E_{\mathrm{zp}}=N S J_{\perp}(\boldsymbol{Q})+\frac{S}{2} \sum_{\boldsymbol{k}} E(h, \boldsymbol{k})
$$

is the zero-point energy contribution to the total ground state energy, and $S E(h, \boldsymbol{k})$ is the field-dependent excitation energy of a magnon with momentum $\boldsymbol{k}$, which is of the form

$$
\begin{aligned}
& E(h, \boldsymbol{k})=\left\{\left[A(\boldsymbol{k})-B(\boldsymbol{k}) \cos ^{2} \Theta_{\mathrm{c}}\right]^{2}\right. \\
& \left.=-\left[B(\boldsymbol{k})\left(1-\cos ^{2} \Theta_{\mathrm{c}}\right)\right]^{2}\right\}^{1 / 2}+C(\boldsymbol{k}) \cos \Theta_{\mathrm{c}}
\end{aligned}
$$




$$
\begin{aligned}
& B(\boldsymbol{k})=J_{z}(\boldsymbol{k})-\frac{1}{2}\left[J_{\perp}(\boldsymbol{k}+\boldsymbol{Q})+J_{\perp}(\boldsymbol{k}-\boldsymbol{Q})\right], \\
& C(\boldsymbol{k})=J_{\perp}(\boldsymbol{k}+\boldsymbol{Q})-J_{\perp}(\boldsymbol{k}-\boldsymbol{Q}) .
\end{aligned}
$$

The ordered moment $M$ is the ground-state expectation value of the $z$ component of the spin $\boldsymbol{S}$ in local coordinates and can be expressed as

$$
M=S\left[1-\frac{1}{2 S}\left(\frac{1}{N} \sum_{\boldsymbol{k}} \frac{A(\boldsymbol{k})-B(\boldsymbol{k}) \cos ^{2} \Theta_{\mathrm{c}}}{E(h, \boldsymbol{k})}-1\right)\right]
$$

in units of $g \mu_{\mathrm{B}}$. Due to quantum fluctuations $M<S$ is smaller than in the classical case, except for the ferromagnet, which is an eigenstate of the Hamiltonian.

In a similar way we can express the magnetization $m$ as the $z$ component of the spin $\boldsymbol{S}$ in the global coordinate system, which is equivalent to a projection of the ordered moment onto the direction of the magnetic field. We get

$$
m=S \cos \Theta_{\mathrm{c}}\left[1+\frac{1}{2 S} \frac{1}{N} \sum_{\boldsymbol{k}} \frac{B(\boldsymbol{k})(A(\boldsymbol{k})-B(\boldsymbol{k}))}{A(0) E(h, \boldsymbol{k})}\right],
$$

in units of $g \mu_{\mathrm{B}}$, again up to first order in $1 / S$.

We note that the results presented in this section are in no way specific to the $J_{1 a, b}-J_{2}$ model, but apply to arbitrary spin Hamiltonians on the Bravais lattices, provided the presence of the applied magnetic field does not destroy the U(1) spin symmetry assumed here.

Within the framework of numerical exact diagonalization, the ordered moment, which is strictly speaking a property of the infinite lattice only, can be obtained indirectly from the static structure factor

$$
\begin{aligned}
& S_{N}(\boldsymbol{Q})=\frac{1}{\mathcal{N}} \sum_{i, j=1}^{N}\left\langle\boldsymbol{S}_{i} \boldsymbol{S}_{j}\right\rangle \mathrm{e}^{\mathrm{i} \boldsymbol{Q}\left(\boldsymbol{R}_{i}-\boldsymbol{R}_{j}\right)}, \\
& M^{2}(\boldsymbol{Q})=\lim _{N \rightarrow \infty} S_{N}(\boldsymbol{Q}),
\end{aligned}
$$

where we set $\mathcal{N}=N(N+1 / S)$, and independently of the long-distance correlation function

$$
\lim _{\left|\boldsymbol{R}_{i}-\boldsymbol{R}_{j}\right| \rightarrow \infty}\left|\left\langle\boldsymbol{S}_{i} \boldsymbol{S}_{j}\right\rangle\right|=\left|\left\langle\boldsymbol{S}_{i}\right\rangle\left\langle\boldsymbol{S}_{j}\right\rangle\right|=M^{2}(\boldsymbol{Q}) .
$$

We parameterize the exchange constants according to

$$
\begin{aligned}
& J_{1 a}=\sqrt{2} J_{\mathrm{c}} \cos \phi \cos \theta, \quad J_{1 b}=\sqrt{2} J_{\mathrm{c}} \cos \phi \sin \theta, \\
& J_{2}=J_{\mathrm{c}} \sin \phi, \quad J_{\mathrm{c}}=\sqrt{\frac{1}{2}\left(J_{1 a}^{2}+J_{1 b}^{2}\right)+J_{2}^{2}},
\end{aligned}
$$

introducing an energy scale $J_{\mathrm{c}}$, a frustration angle $\phi$ and an anisotropy angle $\theta$ (not to be confused with the canting angle $\Theta_{c}$ ).

\section{Results and discussion}

Figure 1 displays the ground-state energy as a function of the frustration angle $\phi$ for the isotropic $J_{1 a}=J_{1 b}$ case with $\theta=\pi / 4$. The dotted line displays the classical ground-state energy obtained from Eq. (2), the solid line shows the result from linear spin-wave theory, Eq. (4).
Dots denote the values obtained from extrapolating our exact-diagonalization data to the thermodynamic limit. The agreement between linear spin-wave theory and exact diagonalization is remarkably well, apart from the regions around the classical borders of the CAF phase.

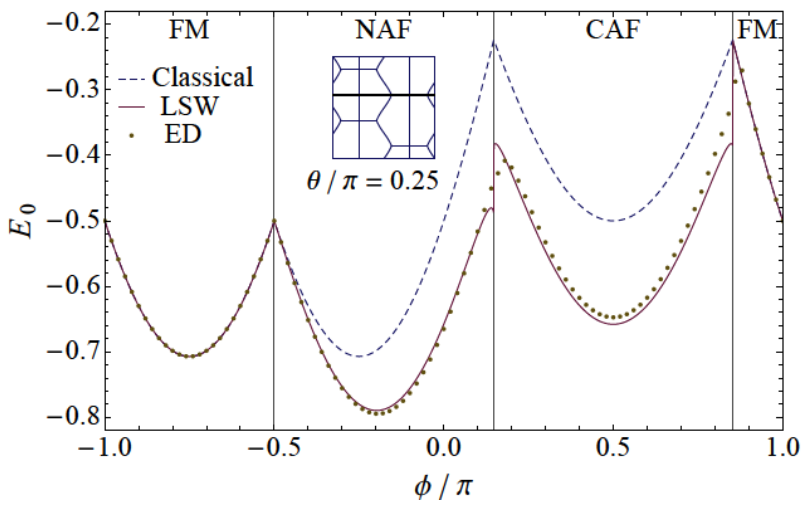

Fig. 1. The ground-state energy as function of the frustration angle $\phi$ for the isotropic model with fixed $\theta=\pi / 4$. The classical energy is shown as dashed line, and the spin-wave results including zero-point fluctuations are presented as solid line. Dots indicate the values for the ground-state energy obtained from extrapolating our exact-diagonalization data. The inset shows a sketch of the classical phase diagram as a function of $\phi$ and $\theta$.

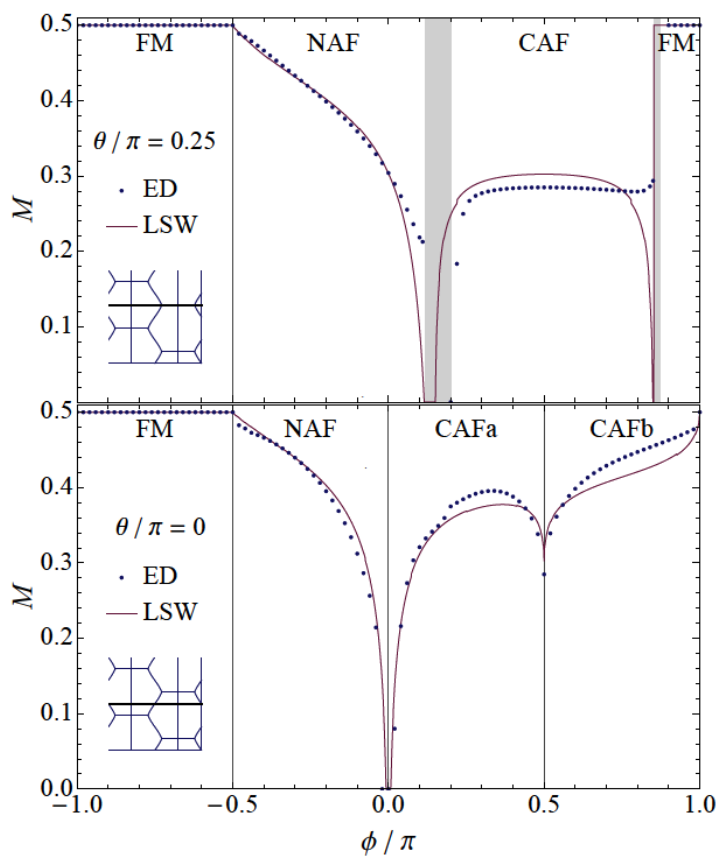

Fig. 2. The extrapolated ordered moment as function of the frustration angle, for (top) the isotropic $\theta=\pi / 4$ case and (bottom) the maximally anisotropic case with $\theta=0$. The gray-shaded areas in the top plot represent the range of frustration angles $\phi$ where the relative error of $M^{2}(\boldsymbol{Q})$ is above 0.1 . 
Figure 2 shows the dependence of the ordered moment on the frustration angle $\phi$, again for the isotropic model with $\theta=\pi / 4$ in the top part, and for the maximally anisotropic model with $\theta=0$ at the bottom. The solid lines denote the results from linear spin-wave theory, Eq. (7), the dots represent the extrapolated values derived from the structure factor according to Eq. (9). Compared to the classical, constant value $M_{\mathrm{cl}}=S=1 / 2$, the ordered moment is strongly reduced due to enhanced quantum fluctuations already at moderate frustration. In fact, $M$ vanishes around the classical borders of the columnar phase, indicating the emergence of two non-magnetic phases not discussed here. However, introducing a spatial anisotropy $(\theta \neq \pi / 4)$ lifts the degeneracy between the two columnar phases $\mathrm{CAFa}$ and $\mathrm{CAFb}$ and stabilizes the ordered moment.

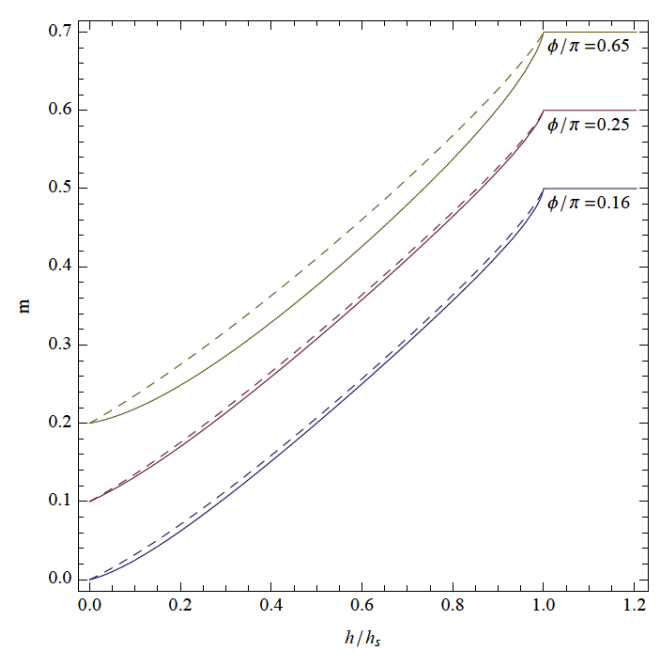

Fig. 3. Uniform magnetic moment $m$ per site as a function of the applied magnetic field $h$ normalized to the saturation field $h_{\mathrm{s}}=2 S A(0)$ at three different frustration angles in the CAF phases, $\phi / \pi=0.16$ (near Néel phase), 0.25 (CAFa), and 0.65 (CAFb near FM). Between each pair of adjacent curves an offset $\Delta m=0.1$ is inserted. The solid lines denote the field dependence in the isotropic case, $\theta=\pi / 4$, the dashed lines denote the maximally anisotropic case, $\theta=0$.

A similar effect can be observed in the field dependence of the uniform magnetization $m$, Eq. (8) shown in Fig. 3 for three different values of the frustration parameter $\phi$. The solid lines in the plot denote $m(h)$ for the isotropic model [13]. In particular, near the crossover to the nonmagnetic regions, the curves are strongly nonlinear, suppressed from the classical linear behavior $m_{\mathrm{cl}}=S h / h_{\mathrm{s}}$ for $h \leq h_{\mathrm{s}}=2 S A(0)$. This effect, which is due to zero-point fluctuations in the ground state, too, is also reduced when introducing a spatial anisotropy, see the dotted lines in the figure.

\section{Conclusions}

To summarize, we have performed an extensive analysis of the $J_{1 a, b}-J_{2}$ model both with linear spin-wave theory and numerical exact diagonalization. The agreement between the two approaches was found to be generally good, and both methods predict the strong suppression and eventual breakdown of the ordered moment in the transition regions at the borders of the columnar phases as a function of frustration. A spatial anisotropy has a stabilizing effect on the ordered moment in the columnar phases.

\section{References}

[1] B. Schmidt, M. Siahatgar, P. Thalmeier, Phys. Rev. B 81, 165101 (2010).

[2] B. Schmidt, M. Siahatgar, P. Thalmeier, Phys. Rev. B 83, 075123 (2011).

[3] E.E. Kaul, H. Rosner, N. Shannon, R.V. Shpanchenko, C. Geibel, J. Magn. Magn. Mater. 272-276, 922 (2004).

[4] N.S. Kini, E.E. Kaul, C. Geibel, J. Phys., Condens. Matter 18, 1303 (2006).

[5] A.A. Tsirlin, H. Rosner, Phys. Rev. B 79, 214417 (2009).

[6] N. Shannon, B. Schmidt, K. Penc, P. Thalmeier, Eur. Phys. J. B 38, 599 (2004)

[7] B. Schmidt, P. Thalmeier, N. Shannon, Phys. Rev. B 76, 125113 (2007).

[8] B. Schmidt, M. Siahatgar, P. Thalmeier, A.A. Tsirlin, J. Phys., Conf. Ser. 200, 022055 (2010).

[9] R.A. Ewings, T.G. Perring, R.I. Bewley, T. Guidi, M.J. Pitcher, D.R. Parker, S.J. Clarke, A.T. Boothroyd, Phys. Rev. B 78, 220501(R) (2008).

[10] R.J. McQueeney, S.O. Diallo, V.P. Antropov, G.D. Samolyuk, C. Broholm, N. Ni, S. Nandi, M. Yethiraj, J.L. Zarestky, J.J. Pulikkotil, A. Kreyssig, M.D. Lumsden, B.N. Harmon, P.C. Canfield, A.I. Goldman, Phys. Rev. Lett. 101, 227205 (2008).

[11] S.O. Diallo, V.P. Antropov, T.G. Perring, C. Broholm, J.J. Pulikkotil, N. Ni, S.L. Bud'ko, P.C. Canfield, A. Kreyssig, A.I. Goldman, R.J. McQueeney, Phys. Rev. Lett. 102, 187206 (2009).

[12] J. Zhao, D.T. Adroja, D.-X. Yao, R. Bewley, S. Li, X.F. Wang, G. Wu, X.H. Chen, J. Hu, P. Dai, Nat. Phys. 5, 555 (2009).

[13] P. Thalmeier, M.E. Zhitomirsky, B. Schmidt, N. Shannon, Phys. Rev. B 77, 104441 (2008). 